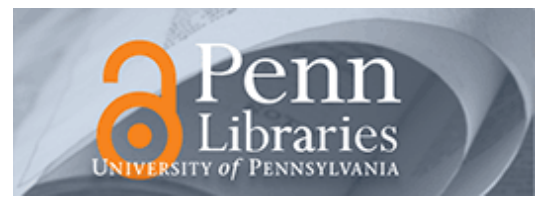

University of Pennsylvania

ScholarlyCommons

1999

\title{
Vanishing Elasticity for Wet Foams: Equivalence With Emulsions and Role of Polydispersity
}

A. Saint-James

University of California, Los Angeles

Douglas J. Durian

University of Pennsylvania, djdurian@physics.upenn.edu

Follow this and additional works at: https://repository.upenn.edu/physics_papers

Part of the Physics Commons

\section{Recommended Citation}

Saint-James, A., \& Durian, D. J. (1999). Vanishing Elasticity for Wet Foams: Equivalence With Emulsions and Role of Polydispersity. Journal of Rheology, 43 (6), 1411-1422. http://dx.doi.org/10.1122/1.551052

At the time of publication, author Douglas J. Durian was affiliated with University of California Los Angeles.

Currently, he is a faculty member at the Physics Department at the University of Pennsylvania.

This paper is posted at ScholarlyCommons. https://repository.upenn.edu/physics_papers/643

For more information, please contact repository@pobox.upenn.edu. 


\title{
Vanishing Elasticity for Wet Foams: Equivalence With Emulsions and Role of Polydispersity
}

\begin{abstract}
We present an experimental study of the rheology of polydisperse aqueous foams of different gas volume fractions $\varphi$. With oscillatory deformation at fixed frequency, we determine the behavior of the maximum stress as a function of the strain amplitude. At low strain, the maximum stress increases linearly, defining a shear modulus G.G. At progressively higher strains, the response eventually becomes nonlinear, defining the yield strain and the yield stress. While $\varphi$ decreases toward $\varphi c=0.635 \pm 0.01, \varphi c=0.635 \pm 0.01$, GG goes to zero, and the yield stress decreases by many orders of magnitude with a quadratic behavior. The yield strain, which can be extrapolated to $0.18 \pm 0.020 .18 \pm 0.02$ at $\varphi=1, \varphi=1$, has a minimum value of $0.045 \pm 0.0100 .045 \pm 0.010$ at $\varphi c . \varphi c$. This behavior shows the occurrence of a melting transition located at $\varphi c, \varphi c$, which can be correlated to the random close packing of spheres. We compare these results to similar ones obtained previously for monodisperse and polydisperse emulsions. Our new experiments clarify the rheological similarities between emulsions and foams, as well as the role of polydispersity. We find that as long as polydispersity is moderate, it does not play a crucial role in the elastic response of foams and emulsions.
\end{abstract}

\section{Keywords}

foams, emulsions, yield stress, elasticity, elastic moduli

\section{Disciplines}

Physical Sciences and Mathematics | Physics

\section{Comments}

At the time of publication, author Douglas J. Durian was affiliated with University of California Los Angeles. Currently, he is a faculty member at the Physics Department at the University of Pennsylvania. 


\title{
Vanishing elasticity for wet foams: Equivalence with emulsions and role of polydispersity
}

\author{
A. Saint-J almes and D. J . Durian \\ UCLA Department of Physics and Astronomy, Los Angeles, \\ California 90095-1547
}

(Received 19 March 1999; final revision received 2 August 1999)

\begin{abstract}
Synopsis
We present an experimental study of the rheology of polydisperse aqueous foams of different gas volume fractions $\phi$. With oscillatory deformation at fixed frequency, we determine the behavior of the maximum stress as a function of the strain amplitude. At low strain, the maximum stress increases linearly, defining a shear modulus $G$. At progressively higher strains, the response eventually becomes nonlinear, defining the yield strain and the yield stress. While $\phi$ decreases toward $\phi_{c}=0.635 \pm 0.01, G$ goes to zero, and the yield stress decreases by many orders of magnitude with a quadratic behavior. The yield strain, which can be extrapolated to $0.18 \pm 0.02$ at $\phi=1$, has a minimum value of $0.045 \pm 0.010$ at $\phi_{c}$. This behavior shows the occurrence of a melting transition located at $\phi_{c}$, which can be correlated to the random close packing of spheres. We compare these results to similar ones obtained previously for monodisperse and polydisperse emulsions. Our new experiments clarify the rheological similarities between emulsions and foams, as well as the role of polydispersity. We find that as long as polydispersity is moderate, it does not play a crucial role in the elastic response of foams and emulsions. (C) 1999 The Society of Rheology. [S0148-6055(99)01206-7]
\end{abstract}

\section{INTRODUCTION}

Aqueous foams are dispersions of gas in a relatively much smaller volume of liquid, stabilized by surfactants absorbed at the liquid-air interfaces [Bikerman (1973); Weaire and Rivier (1984); Aubert et al. (1989); Durian and Weitz (1994); Prudhomme and Khan (1996)]. Foams have very unusual rheological properties: though they are principally made of gas, their rheological properties can be similar to those of a solid, but also to those of a liquid [Kraynik (1988); Gopal and Durian (1995, 1999)]. Indeed, foams can support small shear forces as an elastic solid. However, if the applied stress exceeds a yield stress $\sigma_{y}$, foams flow irreversibly like liquids, and the microscopic structures are no longer similar to the initial ones. The origin of foam elasticity comes from the distortion of bubbles from a perfectly spherical shape, in order to increase their packing density. This packing and the energy stored by the deformation (due to the increase of the surface energy) are responsible for the solid behavior of foams and for the existence of a shear modulus $G$. They are also responsible for the specific geometry of films, Plateau borders and vertices in the limit of dry foams [Plateau (1873)]. Since this packing of the bubble is crucial, the gas volume fraction $\phi$, which describes the packing, is an important parameter. 
One of the most interesting issues is thus to understand if, how, and when the elastic character vanishes with $\phi$ (as in a melting transition). Another interesting issue, specific to yielding materials, remains how to measure the yield stress and yield strain and how to detect the occurrence of nonlinearities in the rheological response. Experimentally, there are no definitive and complete rheological studies of foams, where the gas volume fraction $\phi$ is widely varied and decreased down to the critical value of the melting. Most of the available studies investigate extremely dry foams with $\phi>0.92$ [Wenzel et al. (1970); Khan et al. (1988); Coughlin et al. (1996)]. Even very recently, Gardiner et al. (1998) have performed experiments with a pendulum device to determine the yield stress, but again only dry foams with gas volume fraction bigger than 0.90 have been used. The same is true in the recent work of Zhang et al. (1998). The main reason why there are not yet such complete studies is directly related to the nature of foams: they are out-ofequilibrium materials (subject to drainage, coarsening, and bubble coalescence via film rupture) and are not easy to use, to handle, or to produce uniformly with an initially controlled $\phi$. However, some experimental answers have been given by works on emulsions (dispersion of a liquid into another liquid; oil in water, for instance). In many ways, emulsions are similar to foams, since their static elastic properties are also governed by the packing of bubbles and their distortions. The experimental advantage of emulsions is that they are stable for much longer times than foams. A pioneering work [Princen (1983, 1985); Princen and Kiss (1986a, b)] dealt with polydisperse emulsions of various $\phi$. More recently, experiments have been reported [Mason et al. $(1995,1996,1997)]$ that provide a clear picture of melting for monodisperse emulsions. But, these two studies are in disagreement on many points: the critical value $\phi_{c}$ for the vanishing of elasticity, and the $\phi$ dependence of both the shear modulus $G$ and the yield stress $\sigma_{y}$. These discrepancies, and other irreproducibilities have been ascribed to the role of bubble size distribution. In any case, the strong rheological connections between emulsions and foams are not yet completely proven, and extrapolation to foam rheology may not be so direct. On the theoretical side, foam rheology models have not fully answered these questions of melting transition or polydispersity effects either. Modeling foam rheology is complex and necessarily must be done numerically if one wants to take into account structural disorder and polydispersity. But, the main problem of most of the models is that they are only two dimensional (2D); results for real 3D systems must be extrapolated. In spite of these difficulties, there are some numerical simulations that take into account both disorder and polydispersity. A short review of such models is given by Durian (1997).

We have recently overcome the technical problems of foam production and $\phi$ control [Saint-Jalmes et al. (1999)], and we report here the first comprehensive rheological measurements on polydisperse foams of various gas volume fractions $\phi$. In this article, we focus on the elastic regime, on the yielding, and on how the elasticity vanishes. We present consistent measurements of the yield strain, yield stress, shear modulus, and the critical value $\phi_{c}$. This provides us with a clear picture of the melting transition with $\phi$, and allows us, by comparisons with previous works, to investigate the role of polydispersity in melting and the rheological equivalence between foams and emulsions.

\section{MATERIALS AND RHEOLOGICAL DIAGNOSTICS}

For the following experiments, we used home-made aqueous foams. The production method and the properties of these foams have been described in detail elsewhere [SaintJalmes et al. (1999)]. To summarize, foam is produced via turbulent mixing of gas with a narrow jet of a surfactant solution inside a delivery tube. The gas volume fraction $\phi$ 

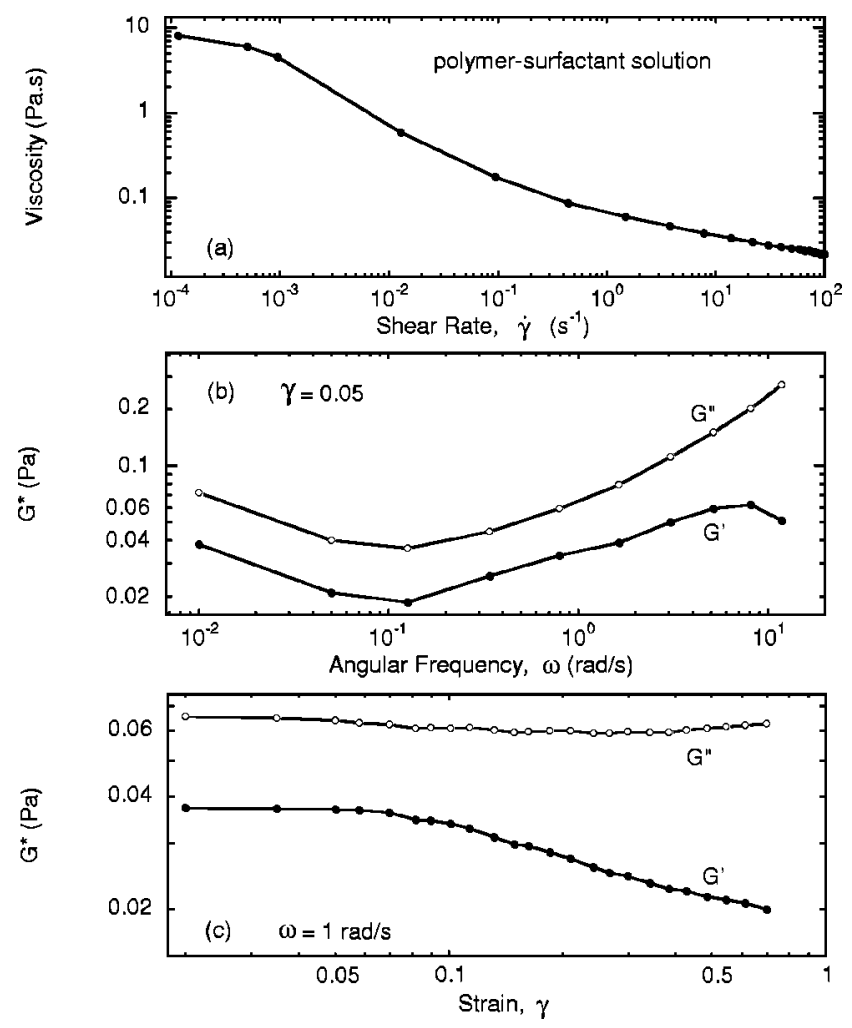

FIG. 1. (a) Viscosity of the aqueous surfactant-polymer solution as a function of the shear rate $\dot{\gamma}$. (b) $G^{\prime}$ and $G^{\prime \prime}$ moduli of the solution as a function of the angular frequency, for $\gamma=0.05$. (c) $G^{\prime}$ and $G^{\prime \prime}$ moduli of the solution as a function of the strain $\gamma$, at $\omega=1 \mathrm{rad} \mathrm{s}^{-1}$.

may be easily varied between $30 \%$ and $99 \%$. The resulting foams have a polydisperse distribution of bubble sizes that is independent of $\phi$. Roughly $60 \%$ of the bubbles have radii between 40 and $70 \mu \mathrm{m}$, with none larger than $100 \mu \mathrm{m}$ or smaller than $10 \mu \mathrm{m}$; the average radius is $R=55 \mu \mathrm{m}$. The aqueous solution used here is based on a recipe realized by Rand (1984): it is a mixture of surfactants [ $\alpha$-olefin sulfonate (AOS), from Witco Corp.], polyacrylic acid polymer (CARBOPOL 941, from BF Goodrich), cosurfactants (dodecanol), solvant (butanol) and water. This solution makes foams which drain extremely slowly because of an increase of both bulk and surface viscosities. Its surface tension is $\sigma=17 \mathrm{mN} \mathrm{m}^{-1}$, as determined by capillary rise. Its rheological properties are summarized in Fig. 1. The solution is shear thinning with a viscosity of a few $\mathrm{Pas}$ at the lowest shear rates [Fig. 1(a)]. As one can see, the viscous contribution to the rheological response is not much higher than the elastic one [Figs. 1(b) and 1(c)], but the typical values of the storage and loss moduli are much smaller than the ones presented below for foams.

The rheological response of our foams is characterized with the UDS 200 rheometer from PAAR-Physica. Two different measurement devices are used, both home built. The first is a Couette cell, with a rotating inner cylinder of diameter $40 \mathrm{~mm}$, length $11 \mathrm{~cm}$, and gap $5 \mathrm{~mm}$. The walls of both cylinders facing the material have been covered with sand paper to avoid wall slipping. The second measurement system is a cone-and-plate device made of transparent acrylic. Here, sand blasting has been used to roughen the walls. The 
diameter of the cone is $127 \mathrm{~mm}$ and the angle is $\beta=10^{\circ}$, meaning that the gap at the edge is around $11.2 \mathrm{~mm}$. These two devices are both used for the experiments as a test for the reproducibility and the validity of the measurements. Foams are directly sent into the gap through the bottom of the outer cylinder for the Couette geometry, or through the middle of the plate for the other geometry. In both cases, foams gently fill all the volume of the cell, without making any inhomogeneities, holes, or gaps. In the cone-and-plate geometry, a vertical wall is necessary to constrain the material in between the cone and the plate (specially when foams are wet). We have found that for reproducibility and for consistency with the Couette device, it is best to put the wall not immediately adjacent to the edge of the cone but rather some distance beyond ( $4 \mathrm{~cm}$ in our case), "a flooded edge." Whatever $\phi$ and the amplitude of the applied deformation, the vertical wall is far enough so that the bubbles at the wall never move. This wall is not necessary for dry foams, and results for these foams with or without the wall are the same. The liquid fractions of foams are measured via a second outlet of the foam production machine, which provides exactly the same foam as the one going into the rheometer cell. So, the same foam is weighed for deducing the actual $\phi$. Since the foam drains with time, we also deduce $\phi$ by measuring the amount of liquid collected beneath the foam after complete drainage. These two methods provide a consistent measurement of $\phi$ with an accuracy better than $1 \%$, free of any initial calibration.

\section{EXPERIMENTS}

\section{A. Types of experiment}

Oscillatory measurements are well suited for studying the rheological response of a material and for detecting the occurrence of nonlinearities and yielding. Actually, two different oscillatory experiments can be performed: amplitude sweep (at fixed frequency) and frequency sweep (at fixed amplitude). We decided to restrict our study to amplitude sweep measurements at fixed frequency for three different reasons. (1) In relation to previous experiments on emulsions [Mason et al. (1995), (1996)], we want to use the same exact approach to provide easy comparisons. (2) Amplitude sweep experiments allow us to study linear and nonlinear responses, and should be sufficient for answering all the melting transition issues; frequency sweep experiments do not provide much insight into these issues. Actually, frequency sweep experiments at fixed amplitude reveal a large range of frequencies where the rheological response is almost constant; thus, the different behaviors collected at a single frequency within that range are also valid for all the other frequencies in that range. As it can be seen in Fig. 2(a), the storage modulus $G^{\prime}$ is almost independent of frequency for frequencies between 0.1 and $10 \mathrm{rad} \mathrm{s}^{-1}$ for the dryer foam; the range of frequency-independent response narrows as $\phi$ decreases. The loss modulus $G^{\prime \prime}$ depends more on frequency, with a minimum around $1 \mathrm{rad} \mathrm{s}^{-1}$ [Fig. 2(b)]. For all foams, at high $\omega$, both $G^{\prime}$ and $G^{\prime \prime}$ increase, as seen and explained previously for emulsions [Liu et al. (1996)]. At low $\omega$, the measurement of $G^{\prime}$ and $G^{\prime \prime}$ requires long runs and is affected by coarsening and drainage (especially for the wettest foams). (3) As just emphasized, coarsening and drainage in foams do not permit low frequency experiments, so all the available information coming from a frequency sweep experiment cannot be extracted. Even with our slow-draining foams, we need to make relatively fast measurements. In the following amplitude sweep experiments, the frequency was fixed at $\omega=1 \mathrm{rad} \mathrm{s}^{-1}$ and the strain amplitude $\gamma$ was swept from $10^{-3}$ to 10. For a minimal number of points, the experimental time is around $5 \mathrm{~min}$. For a very wet foam $(\phi=0.65)$, with the same height as the Couette cylinder, drainage measurements show that less than $4 \%$ of liquid has drained during the first 5 min and that the 


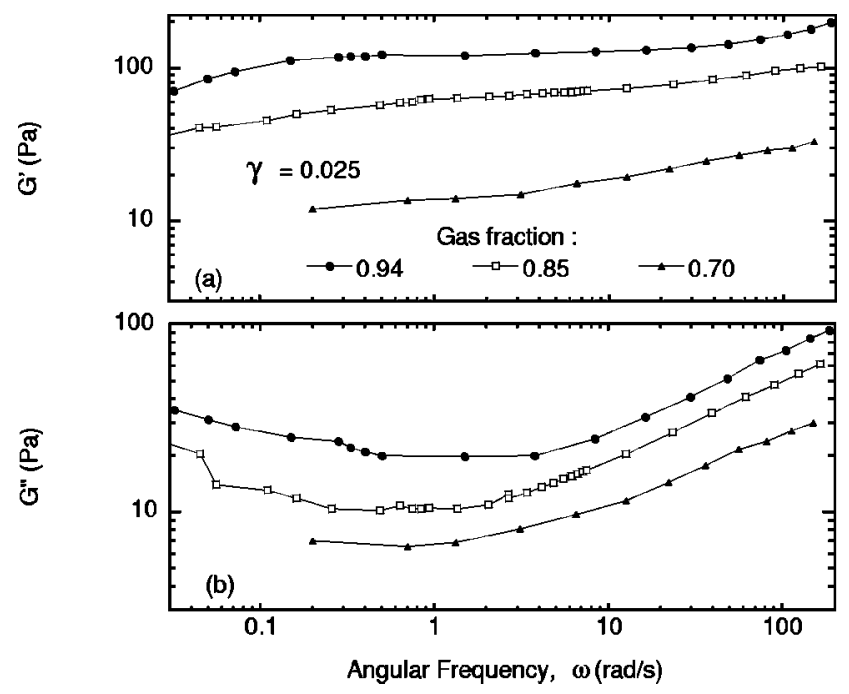

FIG. 2. $G^{\prime}$ and $G^{\prime \prime}$ of foams as a function of the angular frequency $\omega$ for three different gas volume fractions $\phi$. Note the clear plateau behavior at intermediate frequencies, between $0.1 \mathrm{rad} \mathrm{s}^{-1}$ and $10 \mathrm{rad} \mathrm{s}^{-1}$ for the dryer foams. Low frequency measurements cannot be done for $\phi=0.70$ because of drainage. Subsequent experiments are all conducted at $\omega=1 \mathrm{rad} \mathrm{s}^{-1}$.

constant drainage rate obtained during these first minutes is $0.06 \mathrm{~cm} / \mathrm{min}$. This means that at the end of the measurement, a gas fraction gradient has been established on less than the top $3 \%$ of the foam while the rest remains the same. From drainage experiments, foams with $\phi<0.69$ need some "fast" experiments, while drier foams can be studied longer. Amplitude sweep experiments presented below were performed on foams of gas volume fraction $\phi$ between 0.56 and 0.97 made from different surfactant solution batch, with no observed differences from one batch to another.

\section{B. $\gamma$ - dependence results}

Figure 3(a) shows the maximum stress $\sigma_{m}$ as a function of the amplitude of strain $\gamma$. Whatever $\phi$, two different regimes are detected. The first one is a perfectly linear regime where $\sigma_{m}$ is simply proportional to $\gamma$. This could be used to define a shear modulus; however, to be consistent with Mason et al., we will adopt a slightly different definition below. This linear regime is followed by a second, with a sublinear power-law behavior where one can define a nonlinear modulus $G_{\mathrm{nl}}$ and an exponent $\alpha(\alpha<1)$ such as: $\sigma_{m}=G_{\mathrm{nl}} \gamma^{\alpha}$. The intersection between these two regimes defines the yield stress $\sigma_{y}$ and the yield strain $\gamma_{y}$ of a foam at any gas volume fraction $\phi$. Note that linearity in the first regime is achieved only asymptotically as $\gamma \rightarrow 0$, so this is a pragmatic approach to define yielding, as advocated previously [Mason et al. (1996)]. Our moduli and yielding results are independent of the geometry used; however, we must note that there is a small effect due to the measurement device: the crossover between linear and nonlinear regimes is sharper with the cylinders than with the cone and plate. It also appears that this effect is more pronounced for the wettest foams. Nevertheless, the independence of the results with the geometry used demonstrates that slippage or drainage effects are negligible. Furthermore, the height of the sample in the cone-and-plate device is smaller than in the Couette cylinder, so the foam should drain faster [Saint-Jalmes et al. (1999)]; since there are no differences, drainage does not seem to have an effect. Finally, one can see that 

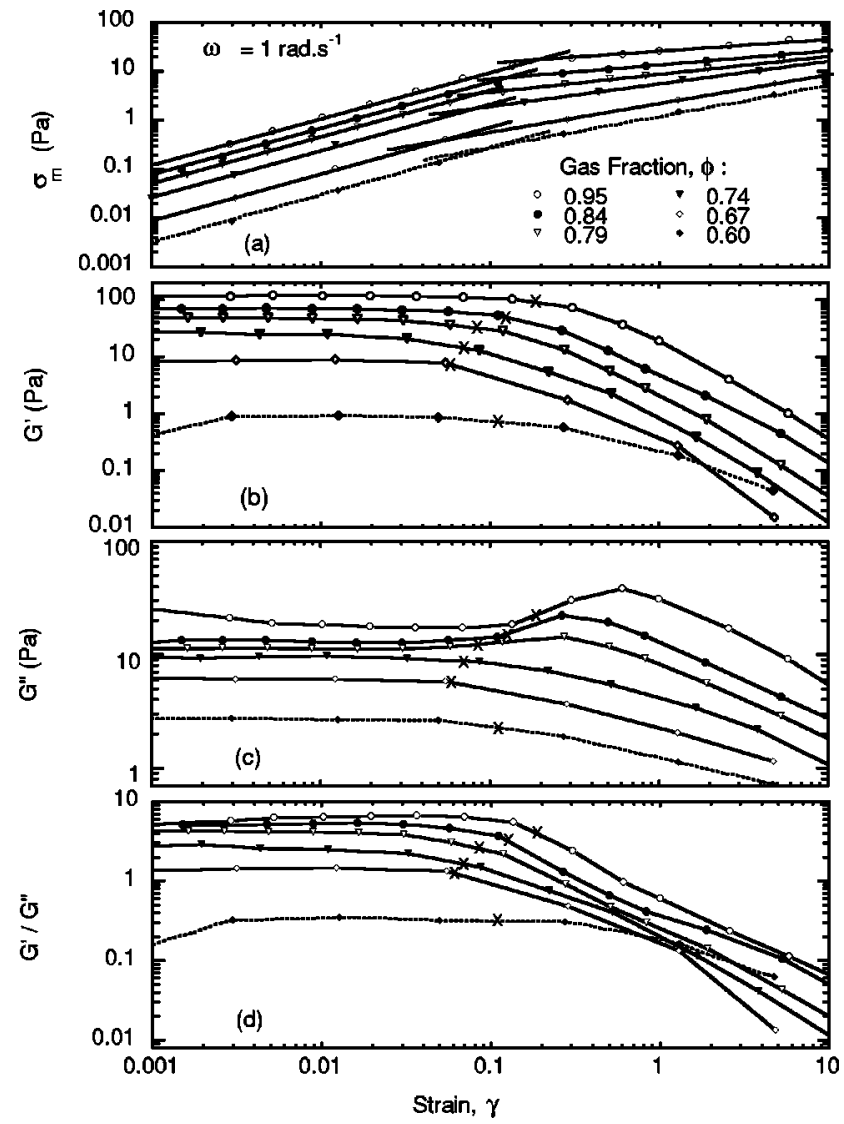

FIG. 3. (a) The maximum stress $\sigma_{m}$ as a function of the strain amplitude $\gamma$, at $\omega=1 \mathrm{rad} \mathrm{s}^{-1}$, for foams of various gas volume fractions $\phi$. The intersection between the first, linear, regime and the second, nonlinear, defines the yield point $\left(\sigma_{y}, \gamma_{y}\right)$. (b) The storage modulus $G^{\prime}$, (c) the loss modulus $G^{\prime \prime}$, and (d) the ratio $G^{\prime} / G^{\prime \prime}$, all as a function of the strain amplitude $\gamma$.

within one experimental run (an amplitude sweep experiment from low strain to high strain is always made with the same foam), there are no departures from the power-law behavior (below or above the yield point) as the points are collected.

For each $\phi$, the yielding can also be seen in the $G^{\prime}$ and $G^{\prime \prime}$ behavior versus strain amplitude at $\omega=1 \mathrm{rad} \mathrm{s}^{-1}$. In Fig. 3(b), $G^{\prime}$ is plotted as a function of $\gamma$ : for each $\phi, G^{\prime}$ is constant at low $\gamma$, and decreases dramatically at high strains, as expected for a material strained beyond its yielding point. The low-strain limit defines the elastic shear modulus of the foam, $G=\lim _{\omega, \gamma \rightarrow 0} G^{\prime}(\omega, \gamma)$, since, (recall from Fig. 1), oscillations at $\omega=1 \mathrm{rad} \mathrm{s}^{-1}$ are already slow enough to give frequency-independent results. Numerically, we find that this is essentially indistinguishable from an alternative definition as $G=\sigma_{m} / \gamma$; even for the very wettest foams, where viscous stresses contribute strongly to $\sigma_{m}$, there is a difference of less than $20 \%$. Here we will adopt the first definition, since it most cleanly separates storage and loss contributions, and since it was also used previously for measurements on monodisperse emulsions [Mason et al. (1995)]. Figure 3(c) shows that, for all foams, $G^{\prime \prime}$ has roughly a constant value at low strain, and decreases at high strains, like $G^{\prime}$. however, for the driest foams, there is a peak located just before the final decrease, and $G^{\prime \prime}$ seems to decrease slowly before that peak. This 
peak gradually disappears, as $\phi$ decreases. We believe that this peak is related to the occurrence of plastic deformations before the yielding. We have reported (with an $X$ ) on these figures, the position of the yield point determined from Fig. 3(a). It appears that the yield strain is located just before the strong decrease of $G^{\prime}$ and just at the foot of the peak for $G^{\prime \prime}$. The meaning of the measured $G^{\prime}$ and $G^{\prime \prime}$ at high strain is complex: they represent the linear response to the strain, but the deformation is nonlinear in this regime, so they are not strictly defined here. However, one can note that $G^{\prime \prime}$ becomes bigger than $G^{\prime}$, meaning that viscous dissipation is bigger than elastic effects, as expected in a irreversibly flowing material. In order to compare these two moduli, Fig. 3(d) shows the ratio $G^{\prime} / G^{\prime \prime}$ for different $\phi$. Beyond the yield strain, for each $\phi$, this ratio decreases dramatically. Note that this ratio is the inverse of the tangent of the phase angle between the applied oscillatory strain and the oscillatory response of the foam.

\section{C. $\phi$ dependence results}

With procedures now established for measuring rheological quantities, we can now turn to the topic of main interest: behavior as a function of increasing liquid content. As can be seen already in Fig. 3, the quantities $\sigma_{y}, \gamma_{y}, G_{\mathrm{nl}}, \alpha, G$ and $G^{\prime} / G^{\prime \prime}$ all depend on the gas volume fraction $\phi$. In Fig. 4, we collect this behavior systematically. Figure 4(a) shows the yield strain behavior: $\gamma_{y}$ has a small variation from roughly $0.18 \pm 0.02$ at $\phi=0.97$ to a minimum value close to $0.045 \pm 0.01$ at $\phi_{c}=0.635 \pm 0.01$. For even lower values of $\phi, \gamma_{y}$ increases again. By contrast, the yield stress has a larger range of variation with $\phi$ [Fig. 4(b)]. When $\phi$ decreases, $\sigma_{y}$ decreases by more than 2 orders of magnitude down to a small constant value. The evolution of $G$ and $G_{\mathrm{nl}}$ with $\phi$ are reported in Fig. 4(c). They both decrease (also by a few orders of magnitude for $G$ ) to almost zero as $\phi$ is decreased from 0.97 . The values of $\phi$ where $G$ and $\sigma_{y}$ reach their small residual constant values (a few Pa for $G$ ) are indistinguishable from $\phi_{c}$, the gas fraction of the minimum value of $\gamma_{y}$ (according to the error bar). The ratio $G^{\prime} / G^{\prime \prime}$ at low strain has a maximum value of 7 for the drier foams, and decreases roughly linearly with $\phi$ [Fig. 4(d)]. It turns out that $G^{\prime} / G^{\prime \prime}$ also reaches a constant value slightly smaller than 1 around $\phi_{c}$. Finally, we found that $\alpha$ increases continuously from 0.2 to 0.8 as $\phi_{c}$ is approached [Fig. 4(d)]. Once again, around $\phi_{c}$, the behavior clearly changes.

\section{INTERPRETATION AND DISCUSSION}

From the $\phi$ dependence of the quantities shown in Fig. 4, we can see in many ways the gradual vanishing of the elasticity of the foams. The physical meaning of such behavior at $\phi_{c}=0.635 \pm 0.01$ can only be identified as the signature of a melting transition. However, we find that $G$ and $\sigma_{y}$ do not fall to exactly zero at $\phi_{c}$, and that there appear to be some elastic properties below $\phi_{c}$. We believe that the residual values of $G$ and $\sigma_{y}$ below $\phi_{c}$ come from the drainage instability. Previous work [Saint-Jalmes et al. (1999)] on drainage has shown that around that same gas fraction, $\phi_{c}$, the drainage is strongly increased, with much higher drainage rates than above $\phi_{c}$. This drainage effect is also confirmed just by direct observation of foams: by eye, it appears that foams with $\phi<0.64$ behave very differently; they simply look like a liquid which flows and spreads very fast. The same drainage problem explains the $\gamma_{y}$ behavior below $\phi_{c}$. So, significant drainage even during the shortest experimental times available creates a significant gas fraction gradient. Part of the sample is below $\phi_{c}$, and has no elasticity; part of it is above $\phi_{c}$, and has a nonzero modulus and yield strain. 

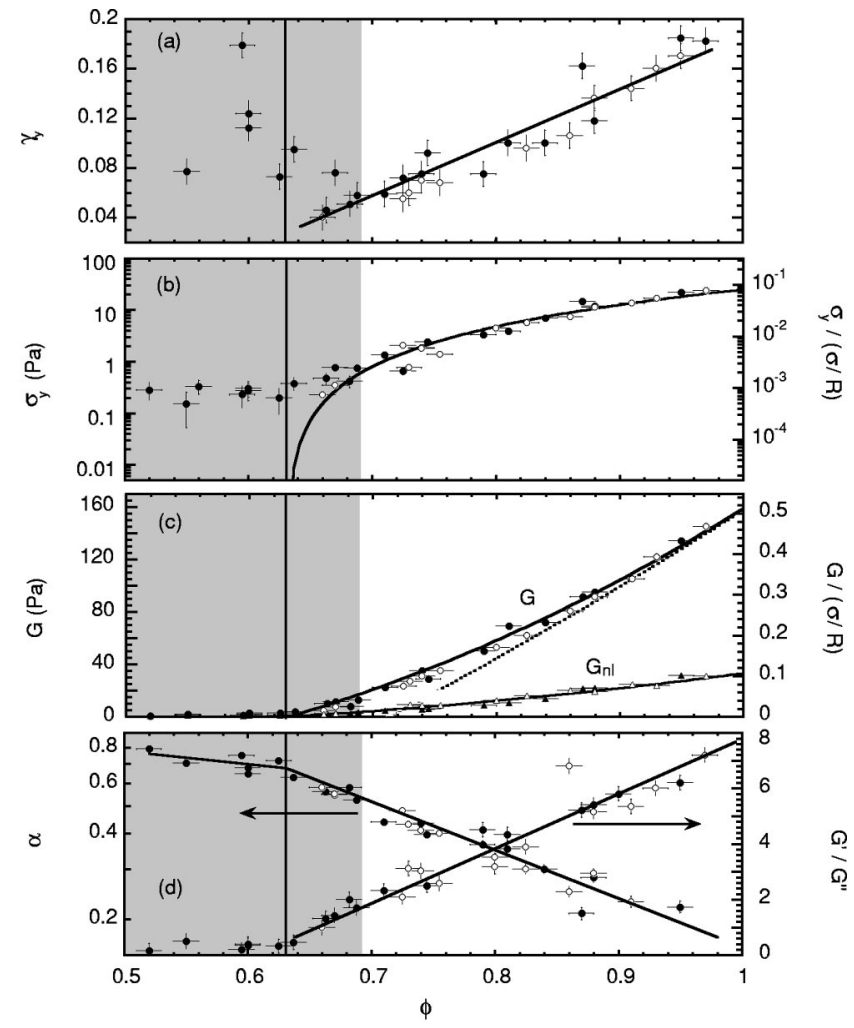

FIG. 4. Dependence of foam rheology on gas volume fraction, $\phi$. (a) $\gamma_{y}$, the yield strain, as a function of $\phi$. $\gamma_{y}$ has a minimum value at $\phi_{c}$, the line is a guide for the eyes. (b) The yield stress $\sigma_{y}$ as a function of $\phi$. The axis on the right represent the value of the yield stress normalized by the Laplace pressure. The solid line represents the results found for monodisperse emulsion (in units of Laplace pressure): $\propto\left(\phi-\phi_{c}\right)^{2}$. (c) The shear modulus $G$ (circles) and the non-linear modulus $G_{\mathrm{nl}}$ (triangles) vs $\phi$. The continuous lines represent the Mason's $\phi$ dependence formula (normalized by $\sigma / R): \propto \phi\left(\phi-\phi_{c}\right.$ ) with $\phi_{c}=0.63$; while the dashed line is Princen's formula $\propto \phi^{1 / 3}\left(\phi-\phi_{c}^{\prime}\right)$ with $\phi_{c}^{\prime}=0.72(\phi>0.75)$. (d) The ratio $G^{\prime} / G^{\prime \prime}$ at low $\gamma$ and the nonlinear exponent $\alpha$ as a function of $\phi$; lines are a guide for the eyes. For all plots: open symbols represent measurements made with the cone and plate device, and closed symbols those made with the Couette cylinders; the shaded area represents the range of $\phi$ where drainage may be a problem $(\phi<0.69)$. The vertical solid line marks the value of $\phi_{c}=0.63$, at which melting occurs.

Outside the regime where drainage artifacts are present, we can compare our results to similar data for monodisperse emulsions [Mason et al. $(1995,1996)]$. The motivation is to track the effects of polydispersity, to test the similarities between foams and emulsions, and to get a more accurate value of $\phi_{c}$. For monodisperse emulsion, $\phi_{c}=0.63$ \pm 0.01 . Our estimation is in perfect agreement: the error bars on the measured values of $\phi_{c}$ in this work and in Mason's work clearly overlap. As already proposed for monodisperse emulsions, we also believe that $\phi_{c}$ is the volume fraction where bubbles are no longer distorted: the foam loses its elasticity as soon as the bubbles attain a spherical equilibrium shape. This picture also explains why drainage effects become very important for foams with $\phi \leqslant 0.64$ : as soon as the bubbles are no longer jammed together, they can move to let the liquid flow vertically very fast through the foam, even transporting the smallest bubbles downward. Note that these two similar values of $\phi_{c}$ found for monodisperse emulsions and in the present study are indistinguishable from $\phi_{\mathrm{rcp}}$, the 
gas fraction of the random close packing of nondeformed spheres [Scott (1960); Bernal and Mason (1960); Gamba (1975); Berryman (1983); Cumberland and Crawford (1987)].

The good agreement with monodisperse emulsion work is confirmed by a few other comparisons. First of all, quantitative and qualitative behavior for $\gamma_{y}$ are extremely similar: we also found a minimum value at $\phi_{c}$ and a roughly linear increase with $\phi$ [shown by the solid line in Fig. 4(a)] up to 0.18 0.02 . Simulations by Kraynik and Reinelt (1996) have provided a value of 0.6 for $\gamma_{y}$ at $\phi=1$ for ordered structures. Structural disorder is responsible for a smaller value by introducing weak regions and allowing local rearrangements of bubbles or groups of bubbles. The fact that $\gamma_{y}$ increases with $\phi$ is due to an increase of order and packing in the material which reduces the number of weak regions [Mason et al. (1996)]. For monodisperse emulsions, where drainage is not as fast, results have been collected below $\phi_{c}$. It has been found that $\gamma_{y}$ increases below $\phi_{c}$ and this has been interpreted in terms of entropic effects [Mason et al. (1996)]. We believe that, in the case of foams, where the bubbles are much bigger, these entropic effects are extremely small and could not be seen (even if drainage were not present).

Next, the $\phi$ dependence of $\sigma_{y}$ agrees also extremely well with the emulsion experiments of Mason et al. (1996). As before, the good agreement is not just qualitative, but also quantitative. In order to make this comparison, we first normalize $\sigma_{y}$ by the Laplace pressure $\sigma / R$; since the elasticity of foams is related to the surface tension $\sigma$ and to the bubble deformation, this is the natural scaling parameter. In our case, $\sigma / R=310 \mathrm{~Pa}$, and we have added on Fig. 4(b) a vertical axis on the right of the figure where $\sigma_{y}$ is normalized by this quantity. In these units, we show the same form empirically used by Mason et al. (1996) for emulsions: $\sigma_{y} \propto\left(\phi-\phi_{c}\right)^{2}$; the result is in excellent agreement with our data [Fig. 4(b)]. Especially for the numerical coefficient: we found a value of 0.53 for the best fit on $\sigma_{y}$ [Fig. 4(b)] while it is 0.52 for emulsions. Furthermore, we can make analogous comparisons for the $\phi$ dependence of the shear modulus $G$. For monodisperse emulsions, it has been proposed that $G$ behaves like $\phi\left(\phi-\phi_{c}\right)$, in units of $\sigma / R$, with $\phi_{c}=0.63$ [Mason et al. (1995)]. In Fig. 4(c), the solid line through the raw data represents the monodisperse emulsion functional form; as one can see, the agreement is quite good. More than simply the functional form, we can again test the quantitative value by looking at the extrapolation at $\phi=1$. For monodisperse emulsions, $G(\phi=1)=0.6$ in units of Laplace pressure; we find here $G(\phi=1)=0.51$. Both results agree with the prediction $G(\phi=1)=0.55$ [Stamenovic (1991); Bolton and Weaire (1992); Reinelt and Kraynik (1996)]. We believe that this small discrepancy is not really significant. It is probably due to missing values at very high $\phi$ for the monodisperse emulsions and due to uncertainty in the Laplace pressure renormalization factor for our work. Note that previous works on very dry foams [Khan et al. (1988); Gardiner et al. (1998)] have also found normalized values of $G(\phi=1)$ close to 0.55 .

In spite of all the similarities between foams and emulsions, we may note one clear difference. The ratio $G^{\prime} / G^{\prime \prime}$ at low strains is quite different from the one for emulsions [Mason et al. (1996)]. In the limit of very dry emulsions, Mason found that $G^{\prime} / G^{\prime \prime}$ reaches a value around 100. We found here a ratio more than 1 order of magnitude smaller (around 7). We can compare the present measurement to others made on foams: Khan et al. (1988) have found a ratio of 5 with home-made foams; recently, Zhang et al. (1998) with commercial Foamy have found a ratio around 10; Cohen-Addad et al. (1998) have also found the same value with the same type of foam. It appears that there is some consistency between all the measurements made on aqueous foams, and a large discrepancy with those on emulsions. Since all these works agree that the storage modulus scales 
with the Laplace pressure and that the normalized extrapolation to $\phi=1$ is around 0.55 , the discrepancy probably comes from the loss modulus and the mechanisms of dissipation in foams.

In spite of that last point, we have surprisingly found that our measurements on the vanishing of elasticity of polydisperse foams are extremely similar to those for monodisperse emulsions. It first appears that rheological similarities between foams and emulsions are strong. Also, regarding the polydispersity issue, our point is that polydispersity does not seem to play a very important role as long as it stays moderate and centered around a single value. We can imagine two different cases where polydispersity could probably change $\phi_{c}$. In the case of an "exotic" type of polydispersity (with a bimodal distribution or with very long tails in the bubble size distribution for instance) one can probably find a higher value of $\phi_{c}$, because small bubbles could fill up voids between larger bubbles and increase the packing. A larger $\phi_{c}$ also occurs if the bubbles are ordered in a crystalline lattice, which increases the efficiency of the packing. Concerning the role of disorder, our experiments have confirmed previous experiments and simulations showing its importance and the differences it produces with ordered systems.

We can only speculate on the reasons why previous experimental works on polydisperse emulsions by Princen and Kiss (1986a) provide different results. Two possible reasons can be advanced: (1) experiments have only been performed with high $\phi$ emulsions $(\phi>0.75)$, (2) empirical functional forms to describe the different behaviors have been deduced directly by analogy with 2D simulations [Princen (1983)] plus a predicted value of $\phi_{c}=0.74$ (hexagonal close packing). The combinations of these points lead to empirical expressions that are only valid in the studied range of $\phi$, and only to extrapolation regarding the value of $\phi_{c}$. For instance, the $\phi$ dependence of $G$ is proposed to be $\propto \phi^{1 / 3}\left(\phi-\phi_{c}\right)$ with $\phi_{c}=0.712$. We have tried such a form for our results [dashed line in Fig. 4(c)] and it appears that this form is good for $\phi>0.82$ (even very good for very dry foams and for the extrapolation at $\phi=1$ ). Nevertheless, it completely misses our experimental results for $G$ at a smaller value of $\phi$. Note that on the study of the shear modulus of polydisperse emulsions, Princen excluded results for the wettest emulsions ( $\phi=0.75$ ), speculating that it would be influenced by drainage [Princen (1983)]. In fact, the value he finds is very close to ours at the same gas fraction. Assuming the validity of this point would lead to a smaller extrapolated value of $\phi_{c}$ in Princen's work, more in accord with our results and those of Mason et al. Some agreement with the Princen experiments is also found for the $\phi$ dependence of $\sigma_{y}$ but only at very high $\phi$ [Princen (1985)]. However, for smaller $\phi$, Princen found $\sigma_{y}$ typically twice smaller than ours.

So far, we have focused on the linear elastic regime of foams. Understanding how foams flow, which dynamical processes are involved, and at which scales, are important issues in the rheology of foams and emulsions. Steady-state shear experiments (when the strain is always bigger than the yield strain) on emulsions have been performed [Mason et al. (1996)] and have provided important insights into the role of fracture for dry emulsions. Using commercial foams, a melting transition at fixed $\phi$ induced by the shear rate $\dot{\gamma}$ has also been reported [Gopal and Durian (1999)]. Their study by diffusing-wave spectroscopy (DWS) has shown the existence of a crossover between a solid-like regime (where the foams flow by discrete rearrangements) to a more liquid-like regime (where the flow is more temporally homogeneous). Here, we also have results above yielding, when foams flow irreversibly $\left[G_{\mathrm{nl}}\right.$ in Fig. 4(c) and $\alpha$ in Fig. 4(d)]. These results are surprising: we find that the dependence of $G_{\mathrm{nl}}$ on $\phi$ has the same form as $G$, both proportional to $\phi\left(\phi-\phi_{c}\right)$ [Fig. 4(c)]. Also, $\alpha$ is definitively not zero for all foams, but 
has a clear dependence on $\phi$. This last point has also been observed by Mason et al. (1995). Regarding this last point, two effects may be responsible. First, we speculate that this increase could reflect the amount and the size of avalanche-like events (or local rearrangements) within the foam. The more the foam is wet, the more avalanche-like events occur in the foam and the more volume is rearranged. Second, the residual stresses after yielding could be purely viscous, and come from the dissipation associated with the motion of the bubbles. Whatever the case, we believe that both the behavior of $\alpha$ and $G_{\mathrm{nl}}$ with $\phi$ reflect some important aspects of the nonlinear flow of foams; this remains to be understood.

\section{CONCLUSION}

This work represents a first set of comprehensive data on foam rheology in which the gas fraction $\phi$ is varied over a wide range. This has been possible by both the use of a new foam production apparatus and of a slowly draining foam formulation. In this article, we have reported rheological evidence of a melting transition in aqueous foams. Both the yield strain and the elastic shear modulus exhibit behaviors which can only be interpreted in such terms. The transition is located at $\phi_{c}=0.635 \pm 0.01$, which we identify as the gas volume fraction $\phi_{\text {rcp }}$ where the bubbles are no longer distorted and are in a random close packing arrangement. From all these measurements, and by comparisons with previous works, it clearly appears that the static elasticity and yielding are very similar in foams and emulsions. We thus provide here direct experimental proof that in such materials the important ingredients are disorder and packing; a moderate polydispersity of the system causes no strong differences from monodispersity (as long as the latter does not cause crystalline ordering).

\section{ACKNOWLEDGMENT}

This work was supported by NASA through Grant No. NAG3-1419.

\section{References}

Aubert, J. H., A. M. Kraynik, and P. B. Rand, “Aqueous foams,' Sci. Am. 254, 74-82 (1989).

Bernal, J. and J. Mason, 'CCoordination of randomly packed spheres,' Nature (London) 188, 910-911 (1960).

Berryman, J. G., 'Random close packing of hard spheres and disks,', Phys. Rev. A 27, 1053-1060 (1983).

Bikerman, J. J., Foams (Springer, New York, 1973).

Bolton, F. and D. Weaire, "The effects of Plateau borders in the two-dimensional soap froth. II. General simulations and analysis of rigidity loss transition," Philos. Mag. B 65, 473-487 (1992).

Cohen-Addad, S., H. Hoballah, and R. Hohler, "Viscoelastic response of a coarsening foam," Phys. Rev. E 57, 6897-6901 (1998).

Coughlin, M. F., E. P. Ingenito, and D. Stamenovic, "Static shear modulus of gas-liquid foam determined by the punch indentation test,' J. Colloid Interface Sci. 181, 661-6 (1996).

Cumberland, D. J. and R. J. Crawford, The Packing of Particles (Elsevier, New York, 1987).

Durian, D. J., “Bubble-scale model of foam mechanics: Melting, non-linear behavior and avalanches,' Phys. Rev. E 55, 1739-1751 (1997).

Durian, D. J. and D. A. Weitz, "Foams," in Kirk-Othmer Encyclopedia of Chemical Technology, 4th Ed., edited by J. I. Kroschwitz (Wiley, New York, 1994), Vol. 11, pp. 783-805.

Gamba, A., "Random close packing of equal spheres,' Nature (London) 256, 521-522 (1975).

Gardiner, B. S., B. Z. Dlugogorski, G. J. Jameson, and R. P. Chhabra, "Yield stress measurements of aqueous foams in the dry limit," J. Rheol. 42, 1437-1450 (1998).

Gopal, A. D. and D. J. Durian, “Nonlinear bubble dynamics in a slowly driven foam,' Phys. Rev. Lett. 75, 2610-2613 (1995).

Gopal, A. D. and D. J. Durian, “Shear induced 'melting' of an aqueous foam,'” J. Colloid Interface Sci. 213, 169-178 (1999). 
Khan, S. A., C. A. Schnepper, and R. C. Armstrong, "Foam rheology. III. Measurements of shear flow properties,' J. Rheol. 31, 69-92 (1988).

Kraynik, A. M., "Foam flows,' Annu. Rev. Fluid Mech. 20, 325-357 (1988).

Kraynik, A. M. and D. A. Reinelt, 'Linear elastic properties of dry soap foams,' J. Colloid Interface Sci. 181, 511-520 (1996).

Liu, A. J., S. Ramaswamy, T. G. Mason, H. Gang, and D. A. Weitz, “Anomalous viscous loss in emulsions," Phys. Rev. Lett. 76, 3017-3020 (1996).

Mason, T. G., J. Bibette, and D. A. Weitz, 'Elasticity of compressed emulsions,'” Phys. Rev. Lett. 75, 20512054 (1995).

Mason, T. G., J. Bibette, and D. A. Weitz, "Yielding and flow of monodisperse emulsions," J. Colloid Interface Sci. 179, 439-448 (1996).

Mason, T. G., M. D. Lacasse, G. S. Grest, D. Levine, J. Bibette, and D. A. Weitz, “'Osmotic pressure and viscoelastic shear moduli of concentrated emulsions,' Phys. Rev. E 56, 3150-3166 (1997).

Plateau, J. A. F., 'Statique Experimentale et Theorique des Liquides Soumis aux Seules Forces Moleculaires" (Gauthier-Villar, Paris, 1873).

Princen, H. M., “'Rheology of foams and highly concentrated emulsions: I. Elastic properties and yield stress of a cylindrical model system,'” J. Colloid Interface Sci. 91, 160-175 (1983).

Princen, H. M., "Rheology of foams and highly concentrated emulsions: II. Experimental study of the yield stress and wall effects for concentrated oil-in-water emulsions,' J. Colloid Interface Sci. 105, 150-171 (1985).

Princen, H. M. and A. D. Kiss, "Rheology of foams and highly concentrated emulsions: III. Static shear modulus,'” J. Colloid Interface Sci. 112, 427-437 (1986a).

Princen, H. M. and A. D. Kiss, "Rheology of foams and highly concentrated emulsions: IV. An experimental study of the shear viscosity and yield stress of concentrated emulsions,' J. Colloid Interface Sci. 128, 176-187 (1986b).

Prud'homme, R. K., and S. A. Khan, eds., Foams: Theory, Measurement, and Application, Surfactant Science Series 57 (Marcel Dekkker, NY, 1996).

Rand, P. B., "Stabilized aqueous foam systems and concentrate and methods for making them," US Patent, 4 442018 (1984).

Reinelt, D. A. and A. M. Kraynik, "Simple shearing flow of a dry Kelvin soap film,' J. Fluid Mech. 311, 327-343 (1996).

Saint-Jalmes, A., M. U. Vera, and D. J. Durian, "Uniform foam production by turbulent mixing: new results on free drainage", European Physical Journal B (to appear).

Scott, G. D., 'Packing of spheres,'” Nature (London) 188, 908-909 (1960).

Stamenovic, D., “A model of foam elasticity based upon the laws of Plateau,' J. Colloid Interface Sci. 145, 255-259 (1991).

Weaire, D. and N. Rivier, "Soap, cells and statistics-random patterns in two dimensions,' Contemp. Phys. 25, 55-65 (1984).

Wenzel, H. G., R. J. Brungraber, and T. E. Stelson, “The viscosity of high expansion foam,” J. Mater. 5, 396-412 (1970).

Zhang, X. D., D. W. Giles, V. H. Barocas, K. Yasunaga, and C. W. Macosko, "'Measurements of foam modulus via a vane rheometer,' J. Rheol. 42, 871-889 (1998). 